\title{
ENERGY CHANGES IN STRESSED BODIES DUE TO VOID AND CRACK GROWTH ${ }^{*}$
}

\author{
J.R.Rice ${ }^{* *}$ and D.C. Drucker ${ }^{* * * *}$
}

ABSTRACT

It is demonstrated that the removal of material or the creation or extension of cracks always reduces the total potential energy of any non-linear or linear stable elastic body under fixed load and displacement boundary conditions. A weaker statement that crack extension releases potential energy in excess of plastic dissipation is established for stable elastic-plastic bodies, with certain strong but reasonable restrictions on the path of traction removal from the new surface. Crack healing, however, always absorbs energy. Implications for the Griffith theory of fracture, including its extension to inelastic materials, and the mechanical aspects of stress corrosion cracking are discussed.

\section{INTRODUCTION}

A comparison is made first between two bodies (a) and (b), each composed of the same distribution of non-linear or linear elastic material, and each in equilibrium under the same loads and displacement boundary conditions, Fig. 1. It is demonstrated that if the voids and cracks of body ( $b$ ) include

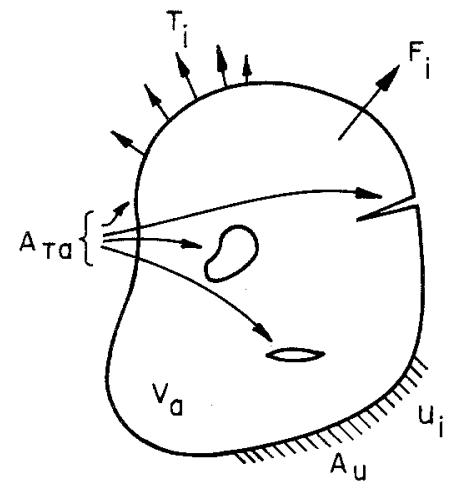

(a)

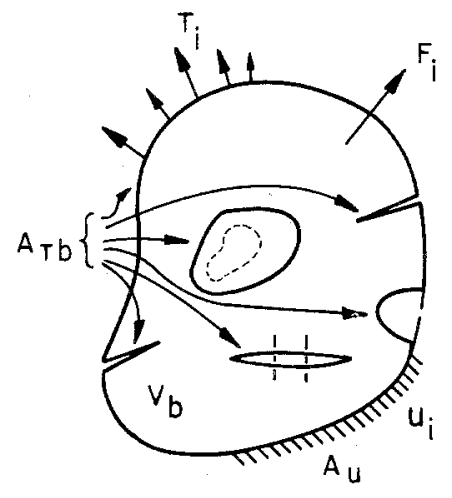

(b)

Fig. 1. Loaded body before (a) and after (b) formation or growth of voids by removal of material or crack extension.

all the voids and cracks of body (a), the total potential energy of the (a) system exceeds or equals that of the (b) system. Creation of new tractionfree surface, whether by fracture or by removal of material, then is shown to release mechanical energy in every loaded or initially stressed elastic body. The corresponding statement for a body of stable elastic-plastic material is much weaker. Crack healing, or the reverse of crack growth, is shown to absorb mechanical energy. But crack growth need release mechanical energy only for a restricted class of paths, which include monotonic reduction of tractions to zero as new crack surface is formed and monotonic slip on discrete surfaces. A discussion is given of the implication of these results for the Griffith ${ }^{1}$ theory of fracture, including its extension to inelastic materials, and for the mechanical aspects of stress corrosion.

* The a uthors acknowledge with their respective thanks a National Academy of Sciences - Nationa1 Research Council postdoctoral fellowship and support by the Advanced Research Projects Agencyunder Contract SD-86 with Brown University. Present manuscript is an expanded and revised version of ARPA Report E 24, August 1965.

* Assistant Professor of Engineering, Brown University.

L. Herbert Ballou University Professor, Brown University. 
Irwin ${ }^{2}$, Bueckner ${ }^{3}$, and Sanders ${ }^{4}$ have discussed the calculation of energy release rates due to crack extension in linear elastic solids, and have emphasized that Griffith-type fracture criteria, resulting from an overall energy balance, are determined by local conditions at the crack tip. Rice ${ }^{5}$, in a recent study of the extension of the Griffith concept, has pointed out that local conditions govern also in inelastic continua.

\section{MINIMUM POTENTIAL ENERGY THEOREM FOR A STABLE ELASTIC SYSTEM}

A strain energy density function

$$
W\left(\epsilon_{m n}\right)=\int_{0}^{\epsilon_{m n n}} \sigma_{i j} d \epsilon_{i j}>0 \text { for } \epsilon_{m n} \not \equiv 0
$$

exists at each point of an elastic material, with value independent of path in strain space from 0 to $\epsilon_{\mathrm{mn}}$. Linear as well as non-linear behavior is included without restriction to isotropy and homogeneity (we do, however, subsequently assume that geometrical non-linearities in strain-displacement gradient relations are negligible). As is customary and often implicit, the elastic material will be presumed stable in the sense introduced by Drucke ${ }^{6}$. In the small

$$
\mathrm{d} \sigma_{i j} \mathrm{~d} \epsilon_{i j} \geqslant 0
$$

for corresponding increments of stress and strain. Considering two states $(q)$ and $(r)$ and integrating along a straight line in stress space so that $\mathrm{d} \sigma_{i j}$ has the direction of $\sigma_{i j}-\sigma_{i j}^{(q)}$, inequality (2) implies

$$
\int_{\epsilon_{\mathrm{mn}}^{(\mathrm{q})}}^{\epsilon_{\mathrm{mn}}^{(\mathrm{r})}}\left[\sigma_{i j}-\sigma_{i j}^{(\mathrm{q})}\right] \mathrm{d} \epsilon_{\mathrm{ij}} \geqslant 0 .
$$

This result holds for all paths between $(q)$ and $(r)$ because elastic behavior is path independent. In the one-dimensional cases of simple tension or simple shear, the postulate of stability means that a change in stress causes a change of like sign in strain; the slope of the stress-strain curve is non-negative.

The characterization of a material as elastic and stable, with the further assumption of the absence of geometric, as opposed to constitutive, non-linear effects is sufficient to ensure validity of the minimum potential energy theorem:

$$
\begin{aligned}
P^{t} & =\int_{V} W\left(\epsilon_{m a}^{t}\right) d V-\int_{A_{T}} T_{i}^{t} u_{i}^{t} d A-\int_{V} F_{i}^{t} u_{i}^{t} d V \\
& \leqslant \int_{V} W\left(\epsilon_{m n}^{*}\right) d V-\int_{A_{T}} T_{i}^{t} u_{i}^{*} d A-\int_{V} F_{i}^{t} u_{i}^{*} d V=P^{* *} .
\end{aligned}
$$

Here $u_{i}^{t}, \epsilon_{m n}^{t}$ are the actual or true displacements and strains produced entirely or in part by the surface tractions $T_{i}^{t}$ and body forces $F_{i}^{t}$. The set $\mathrm{u}_{\mathrm{i}}^{*}, \epsilon_{\mathrm{m} n}^{*}$ is any other kinematically admissible displacement and associated strain field satisfying the boundary conditions on $A_{u}$, with $u_{i}^{*}$ reachable from $u_{i}^{t}$ in the sense that $u_{i}^{*}-u_{i}^{t}$ be continuous. Note that $u_{i}^{t}$ and $u_{i}^{*}$ need not separately be continuous; a state of residual stress may exist at zero load. 
In the comparison of $\mathrm{P}^{\mathrm{t}}$ and $\mathrm{P}^{*}$, the integral over the surface area $\mathrm{A}_{\mathrm{T}}$, where tractions are specified, can be replaced by the integral over the entire surface area $A=A_{T}+A_{u}$ because

$$
\int_{A_{u}} T_{i}^{t} u_{i}^{t} d A=\int_{A_{u}} T_{i}^{t} u_{i}^{*} d A .
$$

The proof of (4) is well-known and follows immediately from the equation of virtual work (or alternately, Green's theorem) for any continuous displacement field $u_{i}^{C}$ and associated strain field $\epsilon_{i j}^{C}$,

$$
\int_{A} T_{i}^{E} u_{i}^{C} d A+\int_{V} F_{i}^{E} u_{i}^{C} d V=\int_{V} \sigma_{i j}^{E} \epsilon_{i j}^{C} d V
$$

where $T_{i}^{E}, F_{i}^{E}, \sigma_{i j}^{E}$ is any equilibrium set. Therefore, with the choice of the true set as the equilibrium set and the difference field $u_{i}^{*}-u_{i}^{t}, \epsilon_{m n}^{*}-\epsilon_{m n}^{t}$ as the displacement set,

$$
\begin{aligned}
P^{*}-P^{t} & =\int_{V}\left[W\left(\epsilon_{m n}^{*}\right)-W\left(\epsilon_{m n}^{t}\right)-\sigma_{m n}^{t}\left(\epsilon_{m n}^{*}-\epsilon_{m n}^{t}\right)\right] d V \\
& =\int_{V}\left[\int \epsilon_{m n}^{t}\left(\epsilon_{i j}^{*}-\sigma_{i j}^{t}\right) d \epsilon_{i j}\right] d V \geqslant 0
\end{aligned}
$$

from (3). For elastic materials of the usual type with single valued relations between stress and strain, the equality sign applies in (4) and (6) only when the two states ( $t$ ) and (*) are identical.

\section{CHANGE IN POTENTIAL ENERGY DUE TO QUASISTATIC VOID GROWTH AND CRACK EXTENSION, (a) TO (b), IN ELASTIC MATERIALS}

Consider any loaded elastic body in equilibrium, $T_{i}$ specified on $A_{T a}$, $F_{i}$ specified in $V_{a}, u_{i}$ specified on $A_{u}$, fig. $1 a$. The special case of residual stress under zero external load is included. As indicated schematically, the body may contain voids, notches, and cracks. Suppose that separately or in combination, material is removed from unloaded regions of the body or portions of the boundary, additional cracks and voids are introduced, and existing cracks are extended, fig. 1b, while the given loads and displacements are held fixed. All void and cracks of the initial state (a) are contained in those of the final state (b). Conversely the material volume $V_{b}$ is contained in $\mathrm{V}_{\mathrm{a}}$.

The boundary conditions on the unchanged area $A_{u}$ are the same for (a) and (b). Therefore the displacements $u_{i}^{a}$ and strains $\epsilon_{\mathrm{m}}^{a}$ of the initial state (a) are a kinematically admissible set for the final state (b) in $V_{b}$. From (4), with (a) $\neq(b)$,

$$
\begin{aligned}
P^{b} & =\int_{V_{b}} W\left(\epsilon_{m n}^{b}\right) d V-\int_{A_{T b}} T_{i}^{b} u_{i}^{b} d A-\int_{V_{b}} F_{i}^{b} u_{i}^{b} \\
& <\int_{V_{b}} W\left(\epsilon_{m n}^{a}\right) d V-\int_{A_{T b}} T_{i}^{b} u_{i}^{a} d A-\int_{V_{b}} F_{i}^{b} u_{i}^{a} d V
\end{aligned}
$$


in which the symbol $A_{T b}$ denotes integration over the portion of boundary of $V_{b}$ where surface tractions are specified. The potential energy of state (a) is

$$
P^{a}=\int_{V_{a}} W\left(\epsilon_{m n}^{a}\right) d V-\int_{A_{T a}} T_{i}^{a} u_{i}^{a} d A-\int_{V_{a}} F_{i}^{a} u_{i}^{a} d V
$$

As $T_{i}^{a}=0$ on all surfaces from which material is removed, $T_{i}^{b}=0$ on the new surfaces thus created, and $T_{i}^{a}=T_{i}^{b}$ elsewhere on $A_{T}$, the surface integrals are identical in (8) and the last line of (7). Then, because $F_{i}^{a} \equiv F_{i}^{b}$ in $V_{b}$,

$$
P^{b}<P^{a}-\int_{V_{a}-V_{b}} W\left(\epsilon_{m n}^{a}\right) d V+\int_{V_{a}-V_{b}} F_{i}^{a} u_{i}^{a} d V,
$$

where $V_{a}-V_{b}$ is the region from which material has been removed in going from (a) to (b). If there is zero or negligible body force in this region. or if the region is of zero volume, as for crack introduction or extension, the potential energy theorem (4) gives

$$
\mathrm{P}^{\mathrm{b}}<\mathrm{P}^{\mathrm{a}}
$$

since $W \geqslant 0$. Potential energy is released by the quasistatic introduction and extension of voids and cracks.

Cutting or material removal by corrosion or etching always causes a body to be a weaker spring. Crack extension always makes potential energy available for absorption in the form of surface energy, as in the original Griffith theory, or modified surface energy as in Irwin's ${ }^{2}$ and Orowan' ${ }^{7}$ extension of this theory $\left(\mathrm{P}^{\mathrm{a}}-\mathrm{P}^{\mathrm{b}}\right.$ is set equal to the energy required for fracture).

Two special cases of (10) are of interest. For loading by displacements only $\left(T_{i} \equiv 0\right.$ on $A_{T}, F_{i} \equiv 0$ in $\left.V\right)$, or for zero external load and a residual stress state, $P=\int_{V} W\left(\epsilon_{m n}\right) d V$ so that

$$
\int_{V_{b}} W\left(\epsilon_{m n}^{b}\right) d V<\int_{V_{a}} W\left(\epsilon_{m n}^{a}\right) d V . \quad \text { (fixed grips) (10a) }
$$

Strain energy is decreased from (a) to (b) for loading by fixed grips or by a residual stress state alone. For loading by forces only $\left(u_{i} \equiv 0\right.$ on $\left.\mathrm{A}_{\mathrm{u}}\right)$ and no residual stress state, $\mathrm{P}=-\int_{\mathrm{V}} \Omega\left(\sigma_{\mathrm{mn}}\right) \mathrm{dV}$ where $\Omega$ is the complementary energy density $\int_{0}^{\sigma_{m n}} \epsilon_{i j} d \sigma_{i j}$. This result follows readily from the principle of virtual work (5). Consequently

$$
\int_{\mathrm{V}_{\mathrm{b}}} \Omega\left(\sigma_{\mathrm{mn}}^{\mathrm{b}}\right) \mathrm{dV}>\int_{\mathrm{V}_{\mathrm{a}}} \Omega\left(\sigma_{\mathrm{mn}}^{\mathrm{a}}\right) \mathrm{dV} \text {. (fixed forces) }
$$

Complementary energy is increased for loading by fixed forces in the absence of residual stresses. For linear elastic systems $\Omega\left(\sigma_{\mathrm{mn}}\right)=\mathrm{W}\left(\epsilon_{\mathrm{mn}}\right)$ so that strain energy may either decrease or increase depending on the method of loading, but the total potential energy must decrease. The potential energy 
reduction in void and crack growth may be calculated explicitly from (7), (8), and virtual work:

$$
\begin{aligned}
P^{a}-P^{b} & =\int_{V_{a}-V_{b}} W\left(\epsilon_{m n}^{a}\right) d V+\int_{V_{b}}\left[W\left(\epsilon_{m n}^{a}\right)-W\left(\epsilon_{m n}^{b}\right)-\sigma_{i j}^{b}\left(\epsilon_{i j}^{a}-\epsilon_{i j}^{b}\right)\right] d V \\
& =\int_{V_{a}-V_{b}} W\left(\epsilon_{m n}^{a}\right) d V+\int_{V_{b}}\left[\int_{\epsilon_{m n}^{a}}^{\epsilon_{m n}^{b}}\left(\sigma_{i j}^{b}-\sigma_{i j}\right) d \epsilon_{i j}\right] d V \\
& =\int_{V_{a}-V_{b}} W\left(\epsilon_{m n}^{a}\right) d V+\int_{A_{b}}\left[\int_{u_{m}^{a}}^{a}\left(T_{i}^{b}-T_{i}\right) d u_{i}\right] d A
\end{aligned}
$$

for negligible body force in $V_{a}-V_{b}$ or for $V_{a}-V_{b}=0$ (crack extension). $A_{b}$ is the bounding surface of $V_{b} ; T_{i}=T_{i}^{b}$ on that portion where tractions are prescribed, and $u_{i}=u_{i}^{b}$ where displacements are prescribed, so that the surface integral vanishes everywhere except on $\Delta A_{a b}$, the new surface created in going from $(a)$ to $(b)$. Since $T_{i}^{b}=0$ on $\triangle A$ ab

$$
P^{a}-P^{b}=\int_{V_{a}-V_{b}} W\left(\epsilon_{m r}^{a}\right) d V-\int_{\Delta A_{a b}}\left[\int_{u_{m}^{a}}^{\mathrm{a}} T_{i}^{b} d u_{i}\right] d A ;
$$

the potential energy reduction is the initial strain energy of the removed material plus the negative of the work done by tractions on the new surface in the process of reduction from those values due to the stress field of state (a) to zero. In the special case of quasi-static elastic systems, with completely linear behavior up to and beyond the creation of new surface,

$$
P^{a}-P^{b}=\frac{1}{2} \int_{V_{a}-V_{b}} \sigma_{i j}^{a} \epsilon_{i j}^{a} d V-\frac{1}{2} \int_{\Delta A} T_{i b}^{a}\left(u_{i}^{b}-u_{i}^{a}\right) d A .
$$

Irwin ${ }^{2}$ and Bueckner ${ }^{3}$ arrived at this result for cracks $\left(V_{a}=V_{b}\right)$; Irwin's calculation of the energy release rate ${ }^{2}$ for crack extension in terms of the stress intensity factors for the three modes of crack tip deformation then follows directly. In view of the simplicity of eq. (13), calculations of energy changes involving integrations over the entire volume and surface of the body would appear unnecessarily awkward and difficult because boundaries at infinity 8 , must be treated properly (most mathematical solutions are available only for infinite regions). Indeed, Griffith later reported ${ }^{10}$ an error in his original calculation ${ }^{1}$ due to this difficulty, and some recently reported results ${ }^{9}$ based on infinite body integrations lead to energy release rates which are not positive definite.

It is of interest to note differences in the method of calculation of energy release rates for void expansion as opposed to crack extension. Consider a narrow elliptical void under plane strain with a fixed semi-minor axis $\ell_{0}$ and semi-major axis $\ell$. For an increase $\Delta \ell$ of the semi-major axis, the volume integral in (13) is of the order of $\Delta \ell$. In the surface integral, however, $\mathrm{T}_{i}^{\mathrm{a}}$ differs from $\mathrm{T} \mathrm{i}_{\mathrm{i}}=0$ by order $\Delta \ell$ and $\mathrm{u}_{\mathrm{i}}^{\mathrm{b}}-\mathrm{u}_{\mathrm{i}}^{\mathrm{a}}$ also is of order $\Delta \ell$ as may be verified by an explicit calculation based on the solution for an elliptical hole in an infinite sheet under tension. Thus in the limit, as $\Delta \ell \rightarrow 0$, only the volume integral contributes to $\partial P / \partial \ell$. On the other hand, for a crack of length $2 \ell$, the volume integral is zero and the surface integral is order $\Delta \ell$ (due to the stress singularity). Only the surface integral then contributes to $\frac{\partial P}{\partial \ell}$. One may verify, however, that as the ellipse shrinks to a crack, 
$\ell_{0} \rightarrow 0$, both calculations give the same result. While we have been unable to set precise conditions for more general configurations, it would appear that only the volume integral over the removed material in (12) or (13) contributes in the extension of a void from one member of a family of smooth surfaces to another infinitesimally close member.

QUASISTATIC CRACK EXTENSION, (a) TO (b), IN STABLE TIMEINDEPENDENT ELASTIC-PLASTIC BODIES

The path dependence of the work function $\int_{\epsilon_{\mathrm{mn}}^{\mathrm{a}}}^{\epsilon_{\mathrm{mn}}^{\mathrm{b}}} \sigma_{i j} \mathrm{~d} \epsilon_{\mathrm{ij}}$ for irreversible materials prohibits statements of corresponding generality for time-independent elastic-plastic bodies. Both the change in strain energy density and the energy density lost in plastic deformation are included in the integral. For crack extension alone, $V_{a}=V_{b}=V$, the change in potential energy $P^{b}-P^{a}$ plus the plastic dissipation $\mathrm{D}_{\mathrm{ab}}$ is

$$
\begin{aligned}
P^{b}-P^{a}+D_{a b} & =\int_{V}\left[\int_{\epsilon_{m n}^{a}}^{\epsilon_{m n}^{b}} \sigma_{i j} d \epsilon_{i j}\right] d V-\int_{A_{T b}} T_{i}^{b}\left(u_{i}^{b}-u_{i}^{a}\right) d A \\
& -\int_{V} F_{i}^{b}\left(u_{i}^{b}-u_{i}^{a}\right) d V
\end{aligned}
$$

because $T_{i}^{b}=T_{i}^{a}$ on the portion of the boundary where surface tractions are specified, and $F_{i}^{b}=F_{i}^{a}$ in $V$.

With the choice of $u_{i}^{b}-u_{i}^{a}$ as the displacement field, $\epsilon_{i j}^{b}-\epsilon_{i j}^{a}$ as the strain field, and $\sigma_{i j}^{b}$ as the stress field in the equation of virtual work (5),

$$
\begin{aligned}
\int_{A_{T b}} T_{i}^{b}\left(u_{i}^{b}-u_{i}^{a}\right) d A & +\int_{V} F_{i}\left(u_{i}^{b}-u_{i}^{a}\right) d V=\int_{V} \sigma_{i j}^{b}\left(\epsilon_{i j}^{b}-\epsilon_{i j}^{a}\right) d V \\
& =\int_{V}\left[\int_{\epsilon_{m n}^{a}}^{\epsilon_{m i j}^{b}} \sigma_{i j}^{b} d \epsilon_{i j}\right] d V
\end{aligned}
$$

Therefore -14$)$ may be rewritten as

$$
P^{b}-P^{a}+D_{a b}=\int_{V}\left[\int_{\epsilon_{m n}^{a}}^{\epsilon_{m n}^{b}}\left(\sigma_{i j}-\sigma_{i j}^{b}\right) d \epsilon_{i j}\right] d V
$$

The total release of mechanical energy, by crack extension, over and above the plastic dissipation $D_{a b}$ and the change in stored energy of the body is the negative of (16) or

$$
P^{a}-\left(P^{b}+D_{a b}\right)=\int_{V}\left[\int_{\epsilon_{m n}^{b}}^{\epsilon_{m n}^{a}}\left(\sigma_{i j}-\sigma_{i j}^{b}\right) d \epsilon_{i j}\right] d V
$$

Stability of elastic-plastic material in the large for a path $(q)$ to $(r)$ or (b) to (a) 


$$
\int_{\epsilon_{m n}^{\mathrm{q}}}^{\epsilon \mathrm{r}}\left(\sigma_{i j}-\sigma_{i j}^{\mathrm{q}}\right) \mathrm{d} \epsilon_{i j} \text { or } \int_{\epsilon_{m n}^{\mathrm{b}}}^{\epsilon_{\mathrm{m} n}^{\mathrm{a}}}\left(\sigma_{i j}-\sigma_{i j}^{b}\right) \mathrm{d} \epsilon_{i j} \geqslant 0
$$

does not guarantee a release of mechanical energy, despite the apparent similarity of (17) and (18). The integrals as written in (17) and (18) do go from (b) to (a), but the material of the elastic-plastic body follows the path (a) to (b) during crack extension. This distinction is of no consequence for elastic materials (3) because of their reversibility. Except for special paths, however, it is crucial for elastic-plastic materials which are irreversible.

Fortunately, some of these special paths which guarantee energy release or availability are encountered in practice. If there is no unloading and the path of loading is radial (proportional) or closely so, the distinction vanishes between a proper incremental form of plastic stress-strain relation and a non-linear elastic relation (deformation theory of plasticity). Monotonic slip confined to a set of parallel planes with a perfectly plastic law or a stable work-hardening relation also will fit into this category. So also does any other plastic deformation pattern which simply increases in ratio or nearly in ratio.

In general, however, unloading does occur in some of the regions of the body which have been plastically deformed, directions of principal stresses and maximum shear stresses rotate appreciably, and (18) is not applicable even approximately for paths from (a) to (b).

An alternative expression to (17) in terms of a surface integral then becomes useful. As for (11) and (12), only the newly created crack surface $\Delta \mathrm{A}_{\mathrm{ab}}$ appears because the given loads and displacements are fixed in the transition from (a) to (b)

$$
P^{a}-\left(P^{b}+D_{a b}\right)=-\int_{\Delta A_{a b}}\left[\int_{u_{i}^{a}}^{u_{i}^{b}} \quad T_{i} d u_{i}\right] d A
$$

The form of the integrand $T_{i} d u_{i}$ shows that mechanical energy is released for all crack extension paths from (a) to (b) which obey strong but not unreasonable restrictions. A zero value would be obtained if crack extension along $\Delta A_{a b}$ were to represent an instantaneous and therefore not a quasistatic drop from the high surface traction across the as yet uncracked surface to the zero value for an open crack. If, however, the mechanism is thought of either as one of cleavage with an attractive elastic type of force between the surfaces which separate quasi-statically, or as a ductile opening of voids, $T_{i} d u_{i}<0$ at each stage and energy is released. Any prior plastic deformation as the crack tip approaches the region of incipient fracture contributes to $\mathrm{D}_{\mathrm{ab}}$ but does not affect the surface integral because $\mathrm{du}_{i}$ is zero until the crack begins to open up.

Rice $^{5}$ has discussed the extension of the Griffith concept to fracture in elastic-plastic systems from a point of view which regards plastic energy dissipation away from the immediate surface of separation as an intrinsic part of the constitutive description of a material (as in continuum plasticity theory) rather than as a part of a modified surface energy term ${ }^{2}$. The procedure is analogous to that for an elastic system in which a crack is visualized as extended under various loads and the fracture strength is determined as the load at which the mechanical energy release (from potential energy alone) equals the work (surface energy) not included in the continuum description but required to create new surface. In an elastic-plastic system the mechanical energy surplus is the excess of potential energy change over plastic dissipation and equals the work excluded from the continuum description but required to create new surface. Path limitations which provide such a non-negative mechanical energy surplus do appear physically appropriate (19). This, 
presuming this surplus to be an increasing function of applied load and the non-continuum work to be adequately estimated as the true surface energy for cleavage or the modified surface energy for ductile void growth and separation, the surface energy serves to determine fracture strength in the sense that variations of surface energy for the same fixed constitutive relations cause corresponding variations in fracture strength. Reduction of surface energy by, say, one-half reduces the fracture strength by a comparable amount. The fact that the total plastic dissipation usually is enormous 2,7 compared to the true or the modified surface energy only implies that the surplus is small compared to the dissipation, not that surface energy is inconsequential. It is plausible that a significant reduction in surface energy along the path of crack propagation, with a negligible modification of the constitutive relations, can occur in short-time contact of a material with its environment. Thus embrittlement by contact with liquid metals or similar agents $^{12}$ appears consistent with this extension of the Griffith theory to elasticplastic materials. Williams ${ }^{13}$ has noted a similar dominant role of surface energy in his extension of the Griffith theory to visco-elastic materials.

As already described, the real difficulty in a precise description of the behavior of elastic-plastic bodies lies in the reverse nature of the proof which works so well for elastic bodies. While the system goes from (a) to (b) in (17), the proof goes from (b) to (a) in (3) and (18), or (t) to (*) in (16), an inconsequential difference only for reversible systems. Now consider the reverse process of crack healing, (b) to (a), rather than crack extension. The path integral,

$\int_{\epsilon_{\mathrm{mn}}^{\mathrm{b}}}^{\epsilon_{\mathrm{mn}}^{\mathrm{a}}} \sigma_{\mathrm{ij}} \mathrm{d} \epsilon_{\mathrm{ij}}$ (see (14)), is again the change in strain energy plus the energy dissipated. In analogy to (16) with $D_{b a}$ denoting the total dissipation in crack healing,

$$
P^{a}-P^{b}+D_{b a}=\int_{V}\left[\int_{\epsilon_{\mathrm{mnn}}^{\mathrm{b}}}^{\epsilon_{\mathrm{mnn}}^{\mathrm{a}}}\left(\sigma_{i j}-\sigma_{i j}^{\mathrm{b}}\right) d \epsilon_{i j}\right] d V \geqslant 0
$$

as a proper consequence of stability in the large, because the system now actually traverses the path (b) to (a). Crack healing absorbs energy in all stable time-independent materials; crack extension need not release energy, without path restrictions, except in reversible systems.

Received October 15, 1966.

REFERENCES

1. A.A.Griffith

2. G.R.Irwin

3. H.F. Bueckner

4. J.L. Sanders

5. J.R.Rice

6. D.C.Drucker
"The Phenomena of Flow and Rupture in Solids", Phil. Trans. Roy. Soc. London, A 221, (1921), p. 163.

"Fracture", Handbuch der Physik, vol.6, Springer, Berlin, (1958), p.551, and "Fracture Mechanics", Structural Mechanics (Proc. of First Naval symp. on Struct. Mech., Ed. J. N. Goodier and N.J.Hoff), Pergamon, (1960), p. 557.

"The Propagation of Cracks and the Energy of Elastic Deformation", Trans. A.S.M.E., 80, (A ug. 1958), p. 1225 .

"On the Griffith-Irwin Fracture Theory", J. Appl. Mech., 27, (1960), p. 352.

"An Examination of the Fracture Mechanics Energy Balance from the Point of View of Continuum Mechanics", Proc. Intl' 1. Conf. Fracture, Sendai, Japan, (1965).

"A More Fundamental Approach to Stress Strain Relations", Proc.First Nat. Cong. for Appl.Mech., (June 1951), A.S.M.E., p. 487. 
7. E. Orowan

\author{
8. A.J.M.Spencer \\ 9. J.L. Swedlow \\ 10. A.A.Griffith \\ 11. S.T imoshenko and \\ J. N.Goodier \\ 12. A.R.C.Westwood and \\ M.H. Kamdar
}

13. M.L.Williams and "On the Postulate of Stability of Material in the Mechanics of Continua", Jour. de Mécanique, 3, (Juin 1964), p.235.

"Fundamentals of Brittle Behavior in Meta1s", Fatigue and Fracture of Metals (ed. W.M.Murray), Wiley, (1952), p. 139.

"On the Energy of the Griffith Crack", Int.Jour.Engr.Sci., 3, 1965, p. 441.

"On Griffith's Theory of Fracture", Inter.J.Frac. Mech., 1, 3, (September (1965), p.210-216.

"The Theory of Rupture", Proc. First Int'1, Cong. Appl. Mech., Delft, (1924), p. 55 .

Theory of Elasticity,

"Concerning Liquid Metal Embrittlement, Particularly of Zinc Monocrystals by Mercury", Phil.Mag., 8, 89, (1963), p. 787.

"Initiation and Growth of Viscoelastic Fracture", Proc. Int'1. Conf.Eracture Sendai, Japan, (1965), 2, pp.1111-1138.

RÉSUMÉ - On démontre que tout corps à stabilité élastique linéare ou non linéare, soumis à des conditions définies de mise en charge et de limitation de déplacements, voit deminuer son énergie potentielle totale lorsque l'on procède à une ablation de matiere, ou que se créent ou se propagent des fissures.

En ce qui conserne les corps élastoplastiques stables on établit une proposition plus nuancée aus sujet de la réduction de l'énergie potentielle en excès sur la dissipation plastique, lorsque se propage une fissure; certaines restictions importantes encore que raisonnables sont formulées au sujet de la maniere dont évolue I'état de tension à partir des nouvelles surfaces libres créées. La croissance d'une fissure ne correspond à une réduction de l'énergie potentielle méca nique que pour une classe restreinte de modes possibles. Par contre, à la fermeture d'une fissure correspond toujours une absorbtion d'énergie. On discute la portée de ces considérations sur la théorie de la rupture de Griffith, y compris.l' extension de cette théorie au cas des matériaux inélastiques, er les aspects mécaniques que revet la físsuration par corrosion sous tension.

ZUSAMMENFASSUNG - Es wird demonstriert, dass die Wegnahme von Material oder die Schöpfung bzw. Verlängerung von Rissen immer die totale potentiale Energie irgeneines linearen oder nicht linearen stabilen elastischen Körpers unter bestimmten Belastung und Zuständen der Versetzungsgranze verringert. Eine schwächere Behauptung, dass Rissverlängerung potentiale Energie im Übermass zur plastischen Körper festgelegt; mit gegewissen strengen, vernïnftigen Begrenzungen auf den Weg der Zugkraftentfernung von der neuen Oberfläche. Rissheilung aber nimmt immer Energie auf. Tiefere Sinne der Griffithschen Bruchtheorie, deren Erweiterung auf unelastische Stoffe eingeschlossen und die mechanischen Erscheinungen der von Spannungsanfressung verursachten Risse werden Besprochen. 